\title{
Socio-demographic and Clinical Profile of Covid-19 in Children
}

\author{
Noman $\mathrm{F}^{1}$, Ahmed $\mathrm{J}^{2}$, Wahab $\mathrm{MA}^{3}$, Hasnat $\mathrm{F}^{4}$, Anne $\mathrm{RT}^{5}$ \\ DOl:https://doi.org/10.3329/jafmc.v16i2.55304
}

\section{Abstract}

Introduction: The corona virus disease 2019 (COVID-19) is a pandemic disease. Infection by COVID-19 in children is not so common but gradually the numbers of children affected with COVID-19 are increasing day by day. The epidemiological importance and clinical pattern in children with COVID-19 is now a challenge to be described by the clinicians.

Objectives: To describe socio-demographic and clinical characteristics of COVID-19 infected children.

Material and Methods: This cross sectional observational study was conducted among 58 COVID-19 children who were admitted in COVID-19 dedicated Kurmitola General Hospital (KGH), Dhaka from March 2020 to July 2020. Their signs, symptoms, sex, age distribution, laboratory results and familial contact were analyzed.

Results: Among 58 most of the children (63.8\%) were in school going age (5-15years). The number of female (39) was more than male (19) and female-male ratio was 1:0.48. Most of the children (75.9\%) came from urban lower middle socioeconomic group. About $84.4 \%$ children were affected from family member. Among all, (8.6\%) patients had no symptoms. Main symptoms of the children were cough $(36.2 \%)$, only fever $(22.4 \%)$, fever with cough (8.62\%) and diarrhoea (5.2\%). About $12.06 \%$ of the children had additional symptoms including sore throat, nasal congestion, fatigue, headache, body ache, vomiting, abdominal pain, convulsion and rash, $6.9 \%$ had respiratory distress and one child came with convulsion. About 12.1\% patients showed lymphopenia, 18.9\% showed leucopenia, 13.8\% patient had neutrophillia. C-Reactive Protein (CRP) and serum ferritin were high in only $12.1 \%$ and $8.6 \%$ cases respectively. There were $29.3 \%$ patients who had normal Chest X-Ray and $58.6 \%$ had mild lesion, $8.6 \%$ had bilateral consolidation and $3.4 \%$ had patchy opacities.

Conclusions: COVID-19 is not so common in children and usually presents with mild form or no sign symptoms clinically and by laboratory analysis. But it is noteworthy that they may play role in transmission of disease.

Key-words: Pandemic, corona virus disease 2019, clinical pattern of COVID-19 in children.

\section{Introduction}

Novel corona virus spread throughout the world from Wuhan since December 2019. On 11 February 2020, WHO declared that the corona virus disease 2019(COVID-19) was caused by novel corona virus $^{1}$. On 12 March 2020, WHO announced that COVID-19 had reached pandemic status ${ }^{2}$. Corona virus are family of enveloped, single stranded zoonotic RNA viruses belonging to the family corona viridae order Nidovirales ${ }^{3,4}$. Corona virus may cause respiratory problem, gastrointestinal problem, severe acute respiratory distress syndrome (ARDS), coagulopathy, multi-organ failure ${ }^{5}$. Server Acute Respiratory Syndrame (SARS) COV-2 is transmitted by direct contact with infected person or by inhalation of droplets by sneezing and coughing of an infected person ${ }^{6}$. Due to close family contact children and old people are very vulnerable for COVID-19. About $56 \%$ of COVID-19 demonstrate clear evidence of transmission through family gathering ${ }^{1,7}$. Vertical transmission from mother to child is not determined by direct data however baby may be cross infected through close contact with mother. No age group is free from COVID-19. A study investigating 8866 cases of COVID-19 reported that the majority of patients were 36 to 65 years of age with only 14 children $<10$ years age diagnosed with disease ${ }^{8}$. Severe COVID-19 disease has 3 stages: Viral stage, the Cytokine storm and ARDS stage that leads to death. The cytokine storm is due to over active immune response and that may be the main cause of mortality ${ }^{6}$.

In case of children the clinical symptoms are milder than adults. Patients may be symptom free or may presents with mild or moderate symptoms. Common presentations are fever and dry cough, fatigue, body ache, nasal congestion, runny nose, sneezing, sore throat, dizziness, diarrhoea, vomiting and abdominal pain. Some children show only cough or diarrhoea and some are asymptomatic carrier $^{1}$ Few children may come with multisystem inflammatory syndrome (MIS), Kawasaki disease, convulsion or respiratory distress ${ }^{9}$. According to clinical characteristics, paediatric cases with COVID-19 can be divided into asymptomatic infection, mild, moderate, severe and critical ${ }^{1,6}$. Asymptomatic, mild and moderate infection comprise over $90 \%$ of all children who have tested positive for COVID-19 with fewer severe and critical cases (5.9\%) compared to adults $(18 \%)^{6,10}$. Milder Infection in children due to under developed immune response and less number of Angiotensin Converting Enzyme-2 (ACE-2) receptors and also less contact with infected person because most of time they reside in home ${ }^{6}$. The

1. Dr Farhana Noman, MBBS, DCH, Senior Consultant of Paediatrics, Kurmitola General Hospital (KGH), Dhaka (Email: drfarhananoman@gmail.com) 2. Brig Gen Jamil Ahmed, MBBS, MPH, MPhil, Director of KGH, Dhaka 3. Lt Col Md Abdul Wahab, MBBS, MD, Associate Professor of Biochemistry, Armed Forces Medical College, Dhaka 4. Dr Ferdousi Hasnat, MBBS, MD, Assistant Professor of Paediatrics, KGH, Dhaka 5. Dr Rifat Taher Anne, MBBS, FCPS, Medical Officer, KGH, Dhaka. 
median age of paediatric cases is 6.7 years ${ }^{6}$ and no sex preponderance were seen ${ }^{6,11}$. In this study, the experience in terms of clinical presentation, demographic data, laboratory analysis and radiological finding of patients were shared.

\section{Material and Methods}

This cross sectional observational study was conducted among 58 COVID-19 children who were admitted in COVID-19 dedicated Kurmitola General Hospital (KGH), Dhaka from March 2020 to July 2020. The inclusion criteria were Patients aged day 1 to 15 years, and all patients confirmed Covid-19 positive by reverse transcription polymerase chain reaction (RT-PCR). Finally 58 patients were enrolled in this study. The medical data was analyzed from previously taken questionnaire for each patient. Demographic data, medical history, contact history was included in the questionnaire. Signs and symptoms including fever, cough, vomiting, diarrhea, abdominal pain, headache, body ache, fatigue, no symptoms, and hospital stay were recorded. Investigation reports were RT-PCR, leukocyte count, total neutrophil count, lymphocyte count, levels of CRP, serum ferritin, CXR were analyzed. Regarding CT chest (milder form of infection patient attendance were not interested to do this job) children with mild disease should not routinely need CT chest imaging in view of high radiation exposure ${ }^{6,12}$. We took written permission from the local authority for the study. A written consent had taken from attendants/Parents as well. Collected data were analyzed by SPSS version 23.

\section{Results}

Total number of positive cases of paediatric age group was 58 . Fig-1 showed percentage of female (67.3\%) \& male (32.8\%). Fig-2 showed age distribution of children which were $<1$ year $(13.8 \%)$, 1-5 year (20.7\%),6-10 years (24.1\%), 11-15 year (41.4\%). Mean age of children was (7.8). Most of children (63.8\%) were school going (5-15 years). It was also found that $75.9 \%$ patients came from Urban areas. From Old Town, Dhaka 25.9\%, Mirpur14. 79\%,Gazipur 10.3\%, Jatrabari 6.9\%, Narayanganj $10.17 \%$, Uttara-Gulshan 3.4\% \& Cantonment Area (Manikdi, Kafrul, Kochukhet) $3.72 \%$. Outside from Dhaka Madaripur 4.6\%, Narail $2.7 \%$, B.Baria $7.4 \%$, Noakhali $5.2 \%$ and other areas $18.6 \%$. Among all patients $59 \%$ belongs to lower-middle income socioeconomic group and $41 \%$ middle income group. About $84.4 \%$ patients had history of contact with COVID-19 positive cases. Fig-3 showed that 8.6\% patients were asymptomatic. Others had symptoms like cough $36.2 \%$, fever $22.4 \%$, fever with cough $8.6 \%$, diarrhoea $5.2 \%$ and $12.1 \%$ patients had additional symptoms including nasal congestion, sore throat, fatigue, headache, myalgia, vomiting, abdominal pain, rash and convulsion, 6.9\% patients had respiratory distress. Covid-19 also associated with chronic diseases, in this study 5.2\% patient had history of chronic diseases like PDA with recurrent UTI, Nephrotic syndrome and pancreatitis. Table-I shows that $18.9 \%$ patient's had leucopenia, $12.1 \%$ lymphopenia and raised CRP, patients had, 3.8\% patient had neutrophillia. There was Serum Ferritin high in only $8.6 \%$ cases. Table-II showed that $29.3 \%$ patients had normal Chest X-Ray and mild lesion $58.6 \%$, bilateral consolidation $8.6 \%$ and $3.4 \%$ patchy opacities. $8.6 \%$ patients needed $\mathrm{O}_{2}$ support. One patient who came with pancreatitis had elevated serum amylase level. Average total hospital stay of patients was 8-10 days.

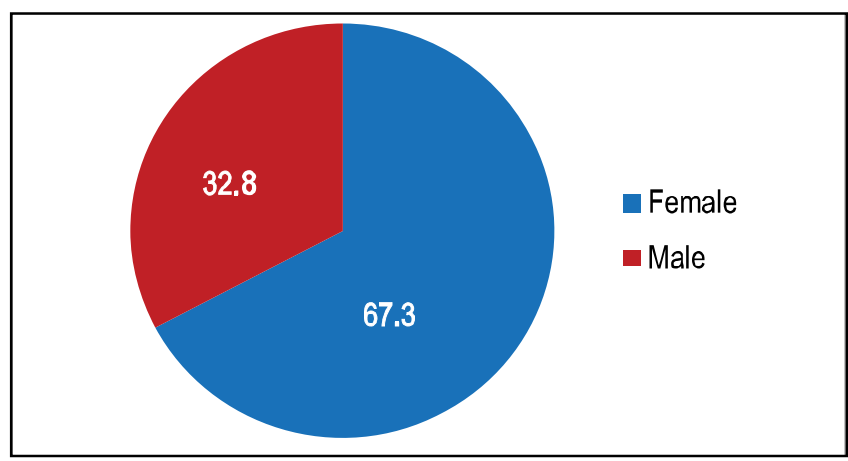

Figure-1: Gender distribution of COVID-19 paediatric patients $(n=58)$

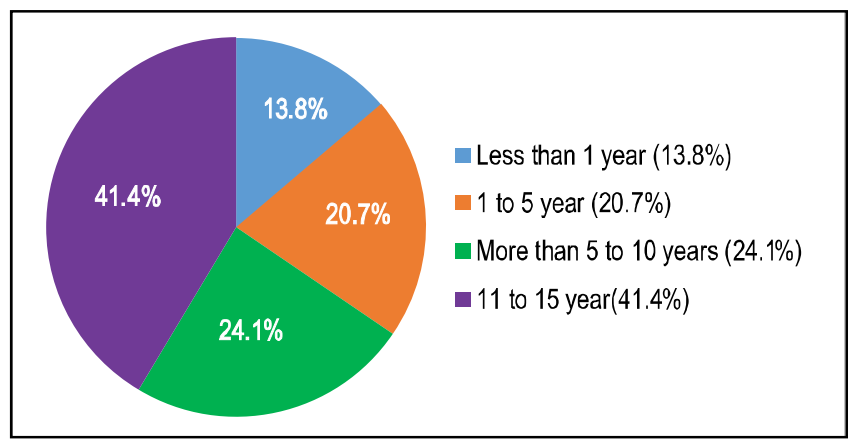

Figure-2: Age distribution of COVID-19 affected children $(n=58)$

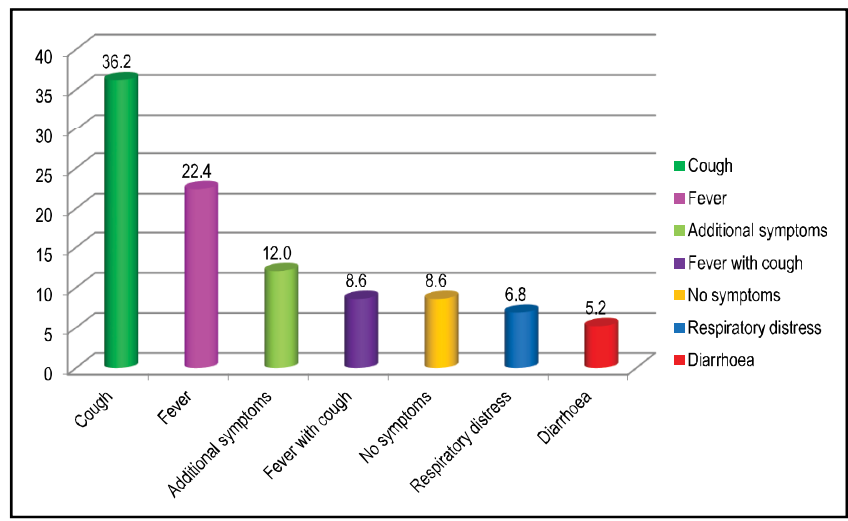

Figure-3: Percentage of clinical symptoms of COVID-19 in Children $(n=58)$

Table-I: Laboratory findings of COVID-19 affected children $(n=58)$

\begin{tabular}{|l|c|c|}
\hline Blood report & Patient number & Percentage \\
\hline Normal leucocyte count & 32 & 55.2 \\
\hline Neutrophillia & 8 & 13.8 \\
\hline Leucopenia & 11 & 18.9 \\
\hline Lymphopenia & 7 & 12.1 \\
\hline C-Reactive Protein increased & 7 & 12.1 \\
\hline Serum Ferritin increased & 5 & 8.6 \\
\hline
\end{tabular}


Table-II: Chest X-Ray findings of COVID-19 infected children $(n=58)$

\begin{tabular}{|l|c|c|}
\hline Chest X-Ray report & Patient number & Percentage \\
\hline Normal chest X-Ray & 17 & 29.3 \\
\hline Mild lesion & 34 & 58.6 \\
\hline BilateralConsolidation & 5 & 8.6 \\
\hline Patchy opacity & 2 & 3.4 \\
\hline
\end{tabular}

Discussion

This cross sectional observational study on paediatric patients was conducted in a COVID-19 dedicated general hospital (Kurmitola General Hospital, Dhaka). We present the clinical and epidemiological characteristics and laboratory analysis of COVID-19 in children. There were 58 patients which was $2.6 \%$ of the total confirmed cases of COVID-19 patients. In china upto 23rd February 77,048 laboratory confirmed cases were reported and $2.1 \%$ were below 19 year of $\mathrm{age}^{13}$. Similar percentage of affected COVID-19 in paediatric group can be found in different studies ${ }^{1,13,14}$.

In Anhui province, China a multi center retrospective cohort study was done in 33 cases COVID-19 patient which was showed positive family contact or family clustering ${ }^{13}$, in this study also showed $84.4 \%$ patients had positive family clustering which were similar to different studies ${ }^{6,15,16}$. In this study, female children were more affected than male, there was no sex preponderance in other studies ${ }^{6,10,17}$.

According to Covid-19 in children and adolescent in Europe, a multinational multicenter cohort study said that 171 PCR confirmed cases in Wuhan suggest about 20\% of children and adolescents with SARS COV-2 were asymptomatic ${ }^{14}$. In this study, $8.6 \%$ children were asymptomatic, whereas it is $15.8 \%$ showed in The New England journal of Medicine and other studies also showed similar obsevations ${ }^{9,18,19}$. One cohort study also showed fever (56\%) and cough (54\%) were the predominant feature ${ }^{14}$. We also found similar type of symptoms in this study. We found additional symptoms which include sore throat, nasal congestion, fatigue, headache, myalgia, vomiting, rash, abdominal pain, convulsion, respiratory distress and diarrhea. In addition to fever and cough many other symptoms were also found in Covid-19 in different studies ${ }^{1,13,14}$.

In children there is relatively less number of patient had lymphopenia and elevated inflammatory marker compared to adults showed in ClinChem Lab Med ${ }^{11,19}$. Summarized from 12 studies on 66 children that $69.6 \%$ had normal leukocytes, $6 \%$ neutropenia, $4.6 \%$ neutrophilia, 3\% lymphopenia and elevated CRP $13.6 \%{ }^{19}$. Regarding investigation from 58 cases of this study normal leukocyte count, leucopenia, lymphopenia, neutrophilia, raised CRP, increased serum ferritin showed in different percentage.

We found having normal Chest X-Ray $29.3 \%$ and mild lesion $58.6 \%$, bilateral consolidation $8.6 \%$ and patchy opacities $3.4 \%$.
Another study showed that among 134 cases, $36 \%$ of the patients had pneumonia, $6.7 \%$ were normal X-Ray and $64.9 \%$ were mild lesion ${ }^{19}$.

\section{Conclusion}

Clinically the symptoms of COVID-19 in children is usually mild in nature. Most of the children were presented with fever, cough and mild pneumonia. Familial contact is the main source of infection in children. Asymptomatic children may not be free from infection when their family members are affected. Due to mild symptoms or asymptomatic infection children may play a role in transmission of virus to community.

\section{References}

1. She J, Liu L, Liu W. COVID 19 epidemic: Disease characteristics in children. wileyonlinelibrary.com/journal/jmv. J Med Virol. 2020:1-8.

2. WHO. WHO characterizes COVID-19 as a pandemic [EB/OL]. Geneva, Switzerland: World Health Organization; 2020.

3. Zimmermann $\mathrm{P}$, Curtis N. Coronavirus Infections in Children Including COVID-19 An Overview of the Epidemiology, Clinical Features, Diagnosis, Treatment and Prevention Options in Children. Pediatr Infect Dis J. 2020; 39:355-68.

4. Fehr AR, Perlman S. Coronaviruses: An overview of their replication and pathogenesis. Methods Mol Biol. 2015; 1282:1-23.

5. Zimmermann P, Nigel C. COVID-19 in children, pregnancy and neonates: A review of epidemiologic and clinical features. Pediatr Infect Dis J. 2020; 39:469-77.

6. Balasubramanian S, Rao NM, Goenka A et al. Coronavirus Disease 2019 (COVID-19) in Children- What We Know So Far and What We Do Not. Indian Pediatr. 2020; 57(5):435-42.

7. Feng F, Xiaoping L. Facing the pandemic of 2019 novel coronavirus infections: The paediatric perspectives. Chin J Pediatr. 2020; 58(2):81-5.

8. Yang Y, Lu QB, Liu MJ et al. Epidemiological and clinical features of the 2019 novel coronavirus outbreak in China. Med Rxiv (PrePrint) 2020. Available at https://doi.org/10.1101/ 2020.02.10. 20021675.

9. Hon KLE, Leung KKY. Paediatric COVID-19: What disease is this? World J Pediatr. 2020; 16(4):323-5.

10. Dong $Y, M o X, H u$ Yet al. Epidemiological characteristics of 2143 paediatric patients with 2019 coronavirus disease in China. J Emerg Med. 2020; 58(4):712-3.

11. Lu X, Zhang L, Du H et al. SARS COV-2 infection in children. N Engl J Med. 2020; 382(17):1663-5.

12. Kelvin AA, Halperin S. COVID-19 in children: The link in the transmission chain. Lancet Infect Dis. 2020; 20(6):633-4.

13. Zhang L, Huang S. Clinical Features of 33 Cases in Children Infected With SARS COV-2 in Anhui Province, China-A Multi-Center 
Retrospective Cohort Study. Front Public Health. 2020; 8:255.

14. Gotzinger F, Santiago-Garcia B, Noguera-Julian A et al. COVID-19 in children and adolescents in Europe, a multinational, multicentre cohort study. Lancet Child Adolesc Health. 2020; 4(9):653-61.

15. Wang $L$, Shi $Y, X i a o T$ et al. Chinese expert consensus on the perinatal and neonatal management for the prevention and control of the 2019 novel coronavirus infection (First edition). Ann Palliative Med. 2020; 8:47.

16. Schwartz DA, Graham AL. Potential maternal and infant outcomes from (Wuhan) coronavirus 2019-nCoV infecting pregnant women: Lessons from SARS, MERS and other human coronavirus infections. Viruses. 2020; 12(2):194.

17. Wei M, Yuan J, Liu $Y$ et al. Novel coronavirus infection in hospitalized infants under 1 year of age in China. JAMA. 2020; 323(13):1313-4.

18. Hu T, Fang $L$, Junling $W$ et al. Clinical characteristics of 2019 novel coronavirus (2019 nCoV) infection in children and family prevention and control. Med J Wuhan Univ. 2020 (PrePrint February 19, 2020).

19. Henry BM, Lippi G, Plebani M. Laboratory abnormalities in children with novel coronavirus disease 2019. Clin Chem Lab Med. 2020; 58(7):1135-8. 